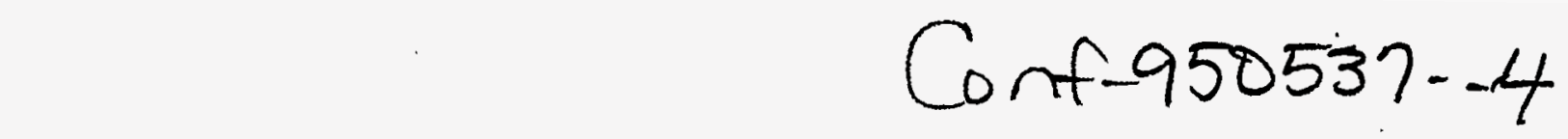

UCRL-JC-118375

PREPRINT

\title{
Experiments and Computer Simulations of the Dynamic Cavity Formed by a Particulated Shaped-Charge Jet in Sand
}

\author{
S. C. Simonson, K. A. Winer, J. E. Reaugh, \\ R. D. Breithaupt, D. W. Baum
}

This paper was prepared for submittal to the 15th International Symposium on Ballistics

Jerusalem, Israel

May 21-24, 1995

February 28, 1995

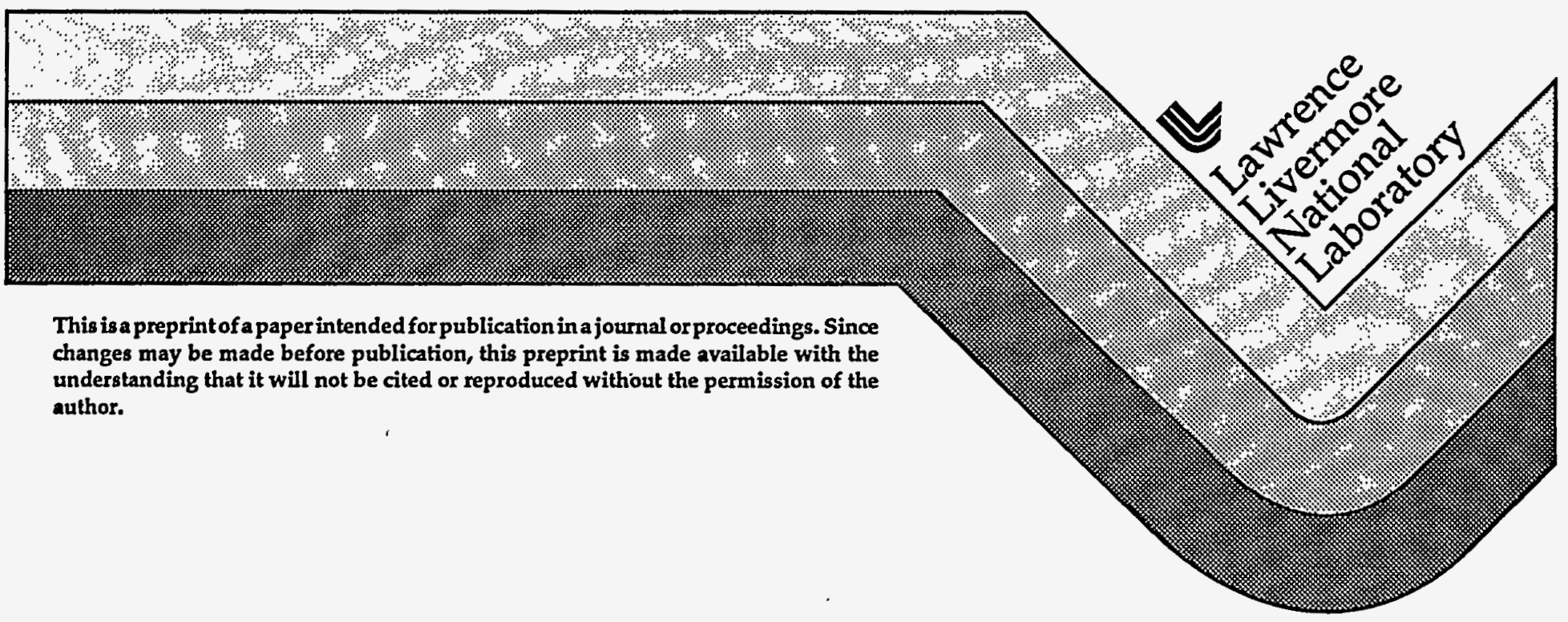

DISTRIBUTION OF THIS DOCUMENT IS UNLIMITEO T5 


\section{DISCLAIMER}

This document was prepared as an account of work sponsored by an agency of the United States Government. Neither the United States Government nor the university of California nor any of their employees, makes any warranty, express or implied, or assumes any legal liability or responsibility for the accuracy, completeness, or usefulness of any information, apparatus, product or process disclosed, or represents that its use would not infringe privately owned rights. Reference herein to any specific commercial products, process, or service by trade name, trademark or manufacturer, or otherwise, does not necessarily constitute or imply its endorsement, recommendation, or favoring by the United States Government or the University of California. The views and opinions of authors expressed herein do not necessarily state or reflect those of the United States Government or the University of California, and shall not be used for advertising or product endorsement purposes. 


\section{DISCLAIMER}

Portions of this document may be illegible in electronic image products. Images are produced from the best available original document. 


\title{
EXPERIMENTS AND COMPUTER SIMULATIONS OF THE DYNAMIC CAVITY FORMED BY A PARTICULATED SHAPED-CHARGE JET IN SAND
}

\author{
S. C. Simonson, K. A. Winer, J. E. Reaugh, R. D. Breithaupt, and D. W. Baum
}

Lawrence Livermore National Laboratory, P.O. Box 808 L-35, Livermore, CA 94551, U.S.A.

Experiments have been carried out to measure the dynamic cavity growth of dry sand during penetration by particulated jets from Viper 65-mm-diameter, $\mathrm{Cu}$-lined conical shaped charges at $1000-\mathrm{mm}$ standoff. The sand target was instrumented with foil switches, piezoelectric pins, and pressure transducers. Flash radiography at $450-\mathrm{keV}$ was used to characterize the jets before impact and to image the target hole during jet penetration. We have developed a dry sand equation of state based on existing Hugoniot data as input to a porous material model incorporated in the 2-D arbitrary Lagrangian-Eulerian hydrocode CALE. We have carried out sand penetration simulations in which the particulated jet is modeled as hot copper rods. By varying parameters in the sand and copper descriptions we identify those features that affect the dynamic cavity formation.

\section{INTRODUCTION}

The design of warheads to attack mines or other deeply buried targets efficiently requires information on the dynamic growth of the cavity that forms around a penetrating projectile. Sand is an inexpensive, readily available material with a mass efficiency against shaped charge jets greater than 1.5 (Held 1987). As such, it is likely to be encountered as overburden in buried target attack. We therefore undertook to study the evolution of the target cavity in dry sand undergoing penetration by a particulated jet. Sequential radiographic images of the target hole coupled with time-of-arrival and pressure data provided a detailed experimental record. To permit better understanding of the phenomena, the experiment was analyzed with a hydrodynamic simulation incorporating a porous sand model.

\section{EXPERIMENTS}

Two experiments were carried out for this purpose. The 65-mm-diameter, Cu-lined Viper precision shaped charge was used at a standoff distance of $1000 \mathrm{~mm}$. The jet from this charge has a tip velocity of approximately $9.5 \mathrm{~km} / \mathrm{s}$. Particulation typically occurs when the tip reaches $800 \mathrm{~mm}$.

In each experiment three $450-\mathrm{keV}$ flash $\mathrm{x}$-ray exposures were made at $10-\mu$ s intervals to record the progress of the jet and the growth of the hole. Experiment 1 (LLNL designation 1410-B9) recorded the jet before impact with the target on two exposures and the initial hole on the third exposure. Experiment $2(1410-B 10)$ recorded the hole development for a second jet on three exposures. The radiographs are reproduced in Figure 1.

The sand target for both experiments was $300 \mathrm{~mm}$ long, $60 \mathrm{~mm}$ high, and $40 \mathrm{~mm}$ wide. The sand used was \#2 grit silicon dioxide, with a measured density of $1.66 \mathrm{~g} / \mathrm{cm}^{3}$. The sides of the 


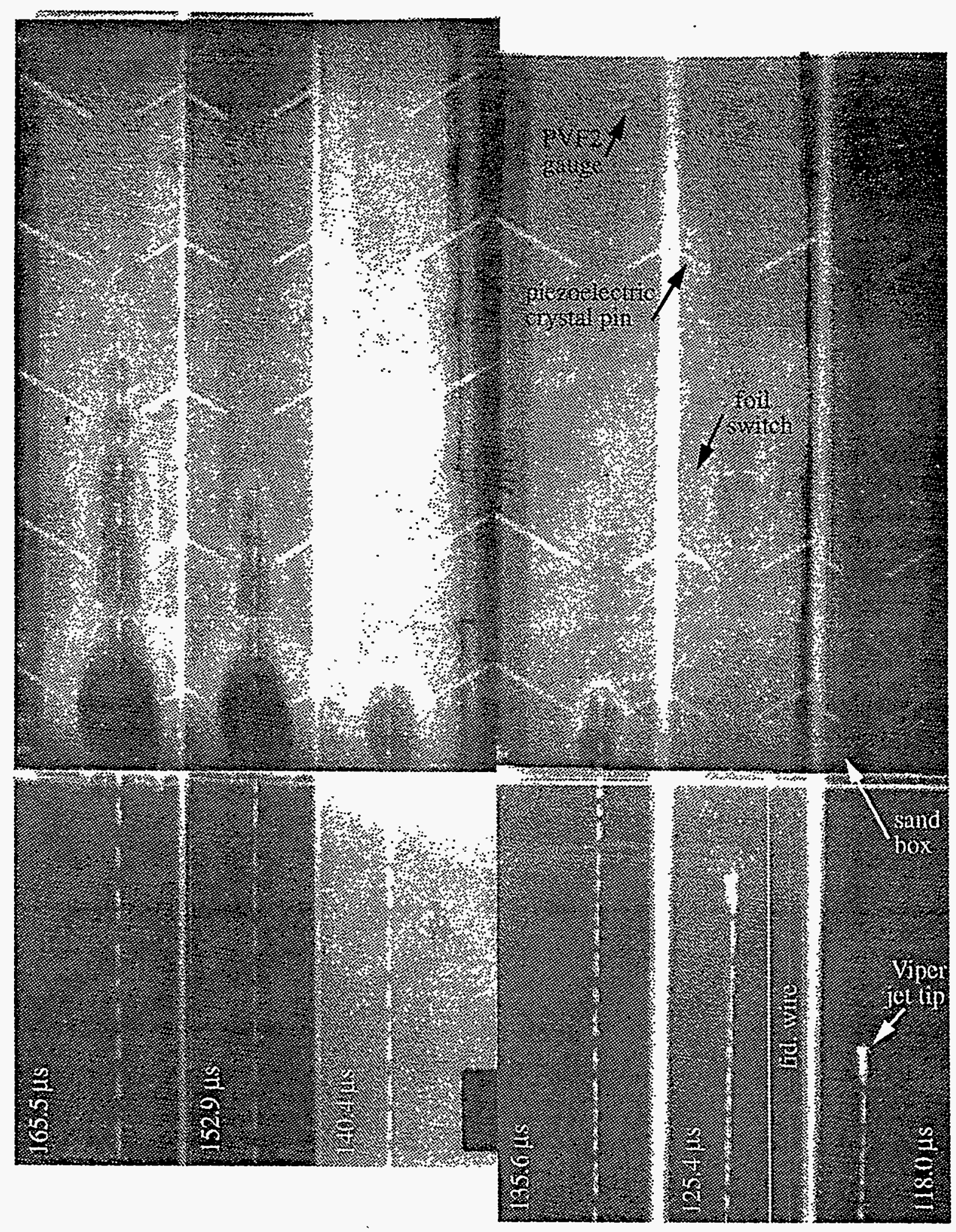

Figure 1. Radiographs from Experiments $1\left(r_{\text {. }}\right)$ and $2(l$.$) . Times of the 450-\mathrm{keV}$ radiographs are indicated, along with locations of piezoelectric crystal pins. foil switches, entrance to the sand box, and approaching Viper jet tip. 
target, through which the radiographs were made, were constructed of 6-mm paper/foam sandwich material. The top and bottom were made of PMMA insulating plastic.

Foil switches formed the entrance and exit membranes, and foil switches were placed every $60 \mathrm{~mm}$ within the target to record the progress of the jet along the experiment centerline. Piezoelectric crystal pin switches were inserted in the sand to measure the time of arrival of the lateral compaction wave. The crystal pins were placed approximately $10 \mathrm{~mm}$ above and below the expected jet trajectory and every $60 \mathrm{~mm}$-along the length of the sand box, starting $20 \mathrm{~mm}$ behind the entrance plane.

In two locations in Experiment 1, polyvinyl difluoride $\left(\mathrm{PVF}_{2}\right)$ pressure transducers were placed against the sides of the box, $20 \mathrm{~mm}$ from the expected jet trajectory. In contrast to the crystal pins, these transducers have the potential for recording the amplitude of the pressure wave.

\section{CALCULATIONS}

$\checkmark$

We developed a dry sand equation of state based on existing Hugoniot data as input to an irreversibly compressible porous material model incorporated in the two-dimensional arbitrary Lagrangian-Eulerian hydrodynamic simulation CALE (Tipton 1995). We then carried out a sequence of sand penetration calculations in which the particulated jet was modeled as axisymmetric, perfectly-aligned, hot copper rods with shapes, separations, and velocities consistent with the sequential radiographs of the Viper jet prior to target impact.

\section{Sand Equation of State}

In CALE there are two standard models that describe the compaction of porous materials. We chose a tabular form, which permits us to mimic the behavior of a model previously developed for the dynamic consolidation of powders (Reaugh 1987). We used that model to fit the measured Hugoniot of dry sand (van Thiel 1977), and extracted points from the compaction curve for the tabular input to CALE. This crush curve (pressure vs. porosity) is shown in Figure 2. The comparison of the model fit with the Hugoniot data is shown in Figure 3.

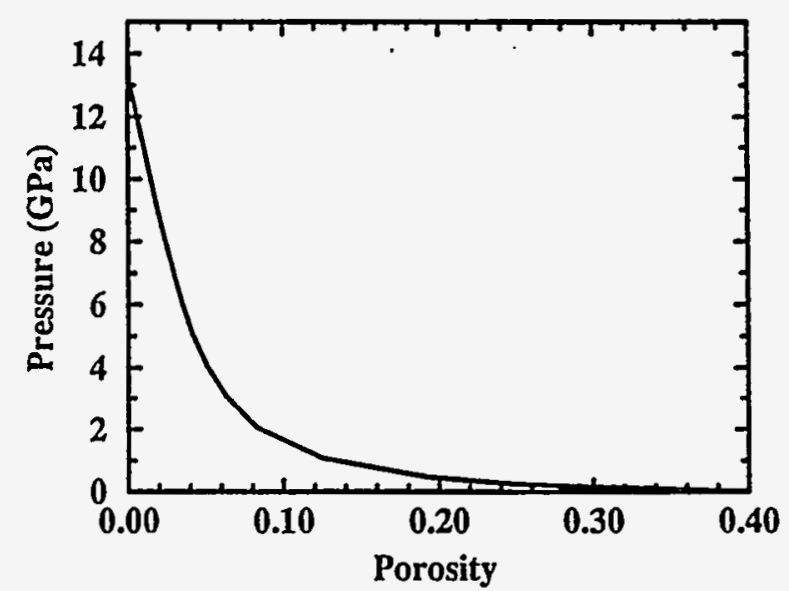

Figure 2. Sand pressure vs. porosity model adopted for CALE calculations.

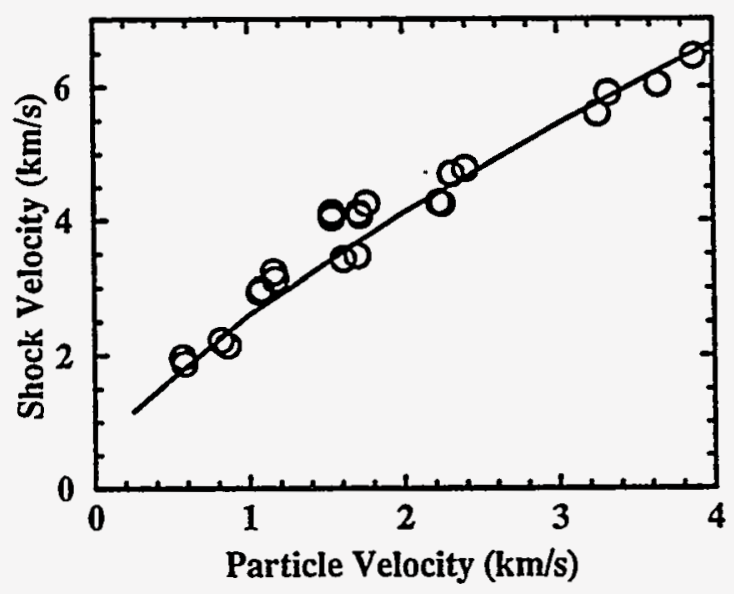

Figure 3. Hugoniot data points for sand, compared with model curve. 
In the CALE calculations, the equivalent stress is taken to be proportional to the pressure (with proportionality constant 1.0) up to a maximum pressure of $2.4 \mathrm{GPa}$, and it is set equal to that value for all higher pressure. The sand has no strength in tension. The maximum shear modulus is $10 \mathrm{GPa}$. Below $2.4 \mathrm{GPa}$ the shear modulus is proportional to the pressure.

When the Hugoniot pressure exceeds the pressure at which all porosity is squeezed out, the details of the pressure-porosity curve do not matter. For our description of sand, this occurs at about $14 \mathrm{GPa}$, which corresponds to a particle velocity of $2 \mathrm{~km} / \mathrm{s}$. Above that pressure any thermodynamically consistent "snowplow" model (Hofmann et al., 1968) will reproduce the Hugoniot. Below that pressure, details of the crush curve will have an effect.

\section{Hydrodynamic Modeling}

The model of the jet used in the CALE calculations is based on the measurements of the particles in the experimental radiographs. The target was represented by a cylinder of sand with a radius $r=27.6 \mathrm{~mm}$, giving the same area as in the rectangular target. The target sides were represented by a lucite shell $4.8 \mathrm{~mm}$ thick. Reflective boundary conditions were applied outside an air zone which was not reached by the expanding target during the course of the calculation. The density of sand was taken to be $1.60 \mathrm{~g} / \mathrm{cm}^{3}$, which is slightly lower than the value of $1.66 \mathrm{~g} / \mathrm{cm}^{3}$ measured. The reference density of silicon dioxide was $2.65 \mathrm{~g} / \mathrm{cm}^{3}$.

CALE was used in a purely Eulerian mode for the penetration calculations. Within the region occupied by the hole, the grid size was $0.3 \mathrm{~mm}$ radially by $0.4 \mathrm{~mm}$ axially. Outside the hole radius the radial grid was allowed to expand gradually.

After the tip particle, the particle radii were all set to $0.9 \mathrm{~mm}$, corresponding to the mean particle diameter of $1.86 \pm 0.26 \mathrm{~mm}$. The lengths were set individually to the nearest full zone occupied by the particle, except that to allow for the narrowed ends of the actual particles, 0.4 $\mathrm{mm}$ was taken off each end of the modeled particles.

The density of the hot copper was taken to be $8.4 \mathrm{~g} / \mathrm{cm}^{3}$, compared with a reference density of $8.93 \mathrm{~g} / \mathrm{cm}^{3}$. This was based on hydrocode simulations of Viper jet formation, together with preliminary calculations using the reference density, which gave too rapid a rate of axial hole growth. The internal energy was set at $0.41 \mathrm{~kJ} / \mathrm{g}$, corresponding to a temperature of $800-1000$ $\mathrm{K}$. For the hot copper, the yield strength was set to an arbitrary low value of $0.1 \mathrm{GPa}$, and the shear modulus to $25 \mathrm{GPa}$. The jet strength plays only a minor role in this calculation.

The hole cavities that resulted from the CALE calculations are shown in Figure 4. The hole profiles are represented as cross sections of density. In each case we see a progression of particles down the axis, which correspond to the particles on the radiographs. Within the sand target we see the compression wave proceeding outward, followed by the growing hole. Pressures were recorded at the locations of the foil switches, the crystal pins, and the $\mathrm{PVF}_{2}$ gauges.

\section{COMPARISON OF SIMULATION AND EXPERIMENT}

\section{Hole Cavity}

The agreement in hole depth between the simulation and the experiments is within $1 \mathrm{~mm}$, or 0.5 percent. This indicates that the choice of relative densities and particle lengths in the simulation was well suited to the experiment. 
The hole cavity, both as simulated with CALE and as observed in the experimental radiographs, develops as a series of scallop-shaped chambers, which are made by the individual

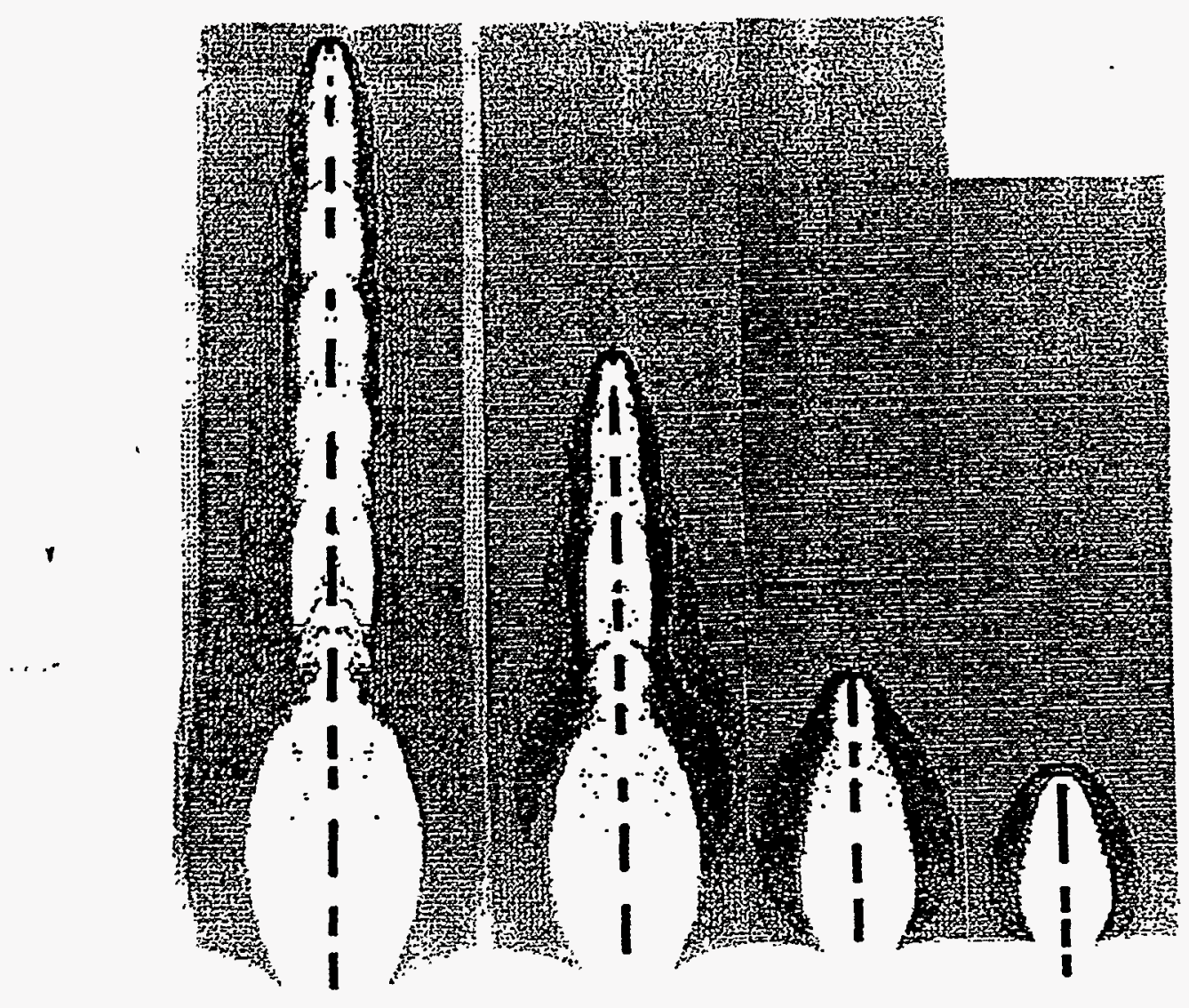

Figure 4. Hole cavities as calculated by CALE for the four sand radiographs shown in Figure 1.

particles. The chambers continue to grow in radius until the kinetic energy delivered by the jet particle is balanced by the work done against the target material.

In a preliminary CALE calculation, the maximum yield strength of the sand was taken to be $2.0 \mathrm{GPa}$. With this value, the calculated maximum radii of the chambers were about $10 \mathrm{per}-$ cent too large for the radiographs in Experiment 2. In an effort to compensate, the maximum yield strength was raised by 20 percent to $2.4 \mathrm{GPa}$ for the calculation shown here. The result was an improvement, and the calculated chamber radii now appear to be too large by only 5 percent.

The actual chamber growth is balanced by energy absorbed by material which experiences a wide range of conditions. Near the axis, a small amount of target material experiences large pressures of about $40 \mathrm{GPa}$, which quickly squeezes out the porosity and arrives on the Hugoniot. Far from the axis at later times much smaller pressures develop which involve larger amounts of material with less well understood energy absorption mechanisms. All of these contribute to determining the radius of the hole. With the simple model adopted here for the sand equation of state, the agreement within 5 percent may be considered good. 
The longer the spacing between particles, the more slowly the material in the narrow part of the hole, between the chambers, moves outward. Consequently, it may remain near enough to the axis to be encountered by later particles and caught up in their wakes. Evidence of this may be seen in the radiographs, particularly in Experiment 2, second and third radiographs (152.9 and $165.5 \mu \mathrm{s})$, at the location of the first foil switch, $60 \mathrm{~mm}$ from the entrance. This is a general phenomenon, seen at many locations, and it is evident in both the simulation and the experiment. It may even cause interference, as will be discussed in the next section.

\section{Foil Switch Timing}

The time of arrival of the jet tip at the locations of the foil switches is shown in Figure 5, for the experiments and for the CALE calculation. The foil switch in Experiment 1 at the entrance

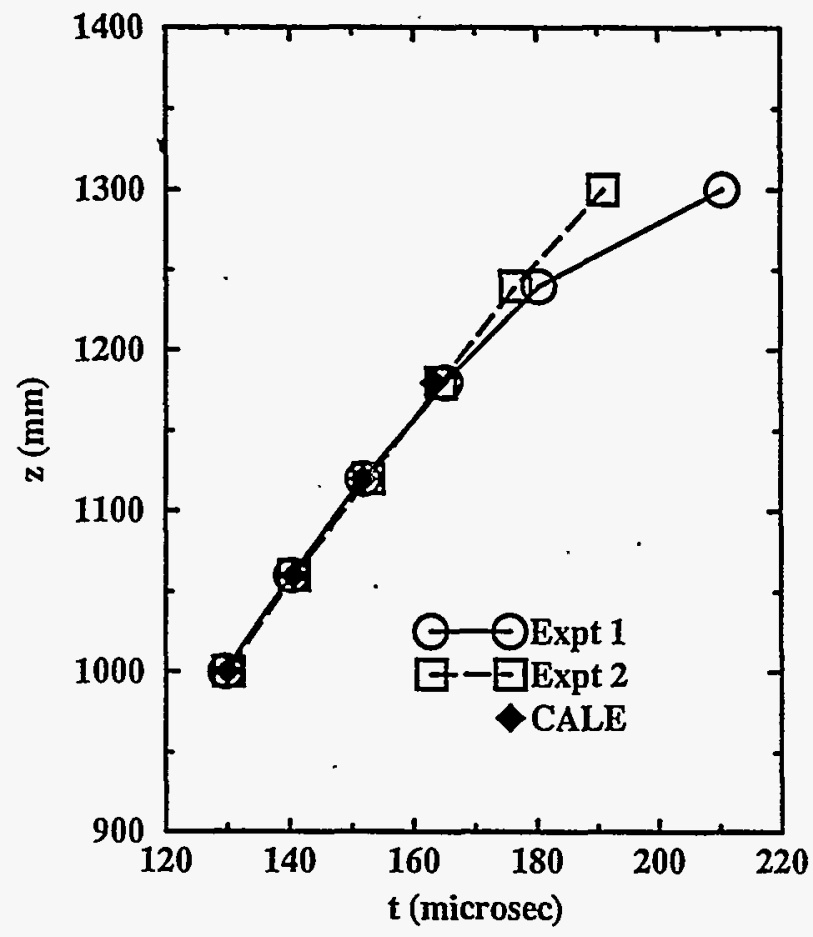

Figure 5. Viper penetration into sand for experiments and CALE calculation.

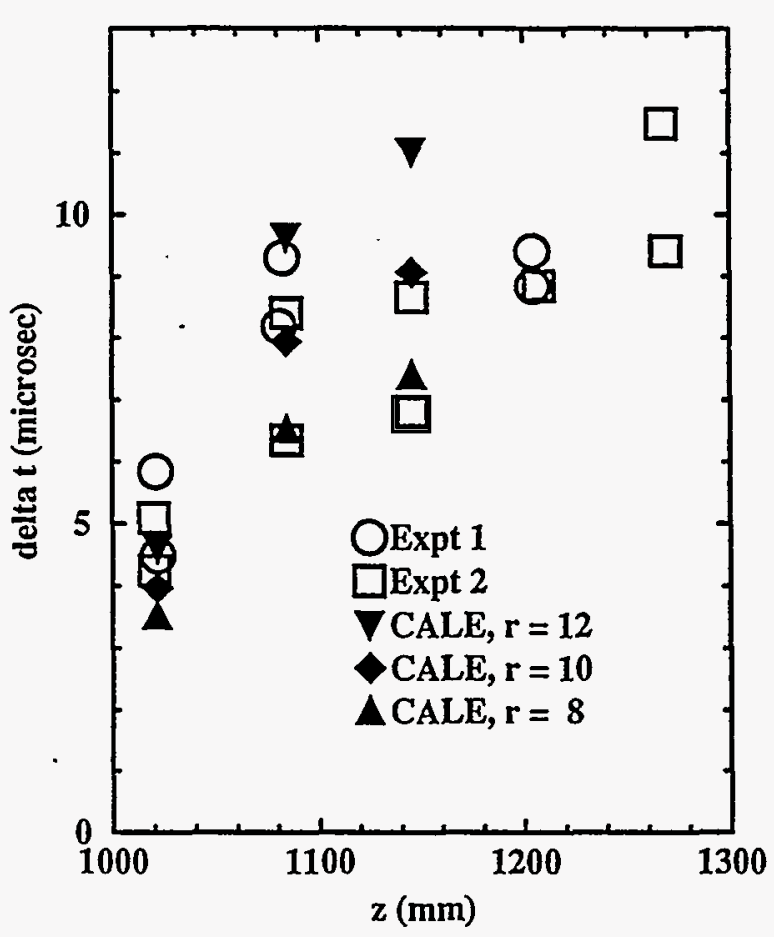

Figure 6. Timing differences between crystal pins and preceding foil switches.

to the target was shorted about $0.16 \mu$ s early by a narrow precursor extending in front of the tip particle, which can be seen in Figure 1. There is evidence that a similar precursor was present at the tip of Experiment 2.

From the entrance switch through the fourth switch, the experiments agree with each other within $\pm 0.65 \mu \mathrm{s}$, and CALE agrees within $\pm 0.68 \mu \mathrm{s}$. At the fifth switch, Experiment 1 falls behind Experiment 2 by $3.77 \mu \mathrm{s}$, and it is late by $19.49 \mu \mathrm{s}$ at the $1300-\mathrm{mm}$ location. This seems to be the result of the bend in the Experiment 1 jet mentioned before, reaching from the tip back to $8.2 \mathrm{~km} / \mathrm{s}$. At a velocity of about $8.2 \mathrm{~km} / \mathrm{s}$, and extending to $7.8 \mathrm{~km} / \mathrm{s}$ (particles no. 15-18), this portion of the jet did not contribute to deepening the hole. Finally, the jet emerged at a velocity of $7.0 \mathrm{~km} / \mathrm{s}$, whereas in Experiment 2 the exiting particle had a velocity of 7.9 $\mathrm{km} / \mathrm{s}$.

The bend in the Experiment 1 jet meant that the first part of the hole was not aligned with the straight part of the jet that came later. This, together with the tendency of target material to 
remain near the axis where there is a large gap between particles, meant that the jet was likely to encounter target material midway down the hole. The largest gaps in the Experiment 1 jet are between particles no. 2 and 3 and between nos. 8 and 9. The target material corresponding to the gap between particles no. 2 and 3 is the likely candidate to have interfered with particles no. 15-18, as it deviates farthest from their trajectory.

\section{Crystal Pin and $\mathrm{PVF}_{2}$ Gauge Timing}

In order to show the propagation of the pressure wave moving outward from the axis, relative timing data are presented in Figure 6. We have plotted the time difference between the arrival of the pressure wave at the pin location and the shorting of the preceding foil switch. The crystal pins were nominally $20 \mathrm{~mm}$ behind the foil switch and $10 \mathrm{~mm}$ from the axis. Actual locations were taken from the radiographs (as projected on the vertical plane). In both experiments, the jet passed closer to one set of pins than to the other. In Experiment 2, the jet's projected displacement was about $2 \mathrm{~mm}$ from the midpoint, as can be seen in Figure 1.

Three sets of locations were recorded in the CALE simulation, at radii of 8,10 , and $12 \mathrm{~mm}$. As the response threshold of the piezoelectric pins is unknown, an arbitrary threshold value of $0.1 \mathrm{GPa}$ was adopted in deriving the CALE times. However, the initial pressure rise occurs as much as $1 \mu$ s earlier for the later points.

The first set of points, near $1020 \mathrm{~mm}$, represents the pressure wave from the tip particle. The propagation time is noticeably shorter than that for the remaining particles. The strength of the wave is larger there (about $4.5 \mathrm{GPa}$ in the calculations $v s .0 .3 \mathrm{GPa}$ for the next location), and the axial offset is a smaller fraction of the particle size. Therefore, the propagation is dominated more by the compression phase and less by the succeeding rarefactions.

Despite these advantages, the first set of data points is hard to analyze. For Experiment 1; the cavity is asymmetrical and the jet tip is offset. Therefore, the delay between the CALE times

and the experimental times may reflect geometrical variances as opposed to equation-of-state differences. For Experiment 2, the exact shape of the tip particle and the projected location of the undisturbed pins was not recorded.

The remaining sets of data, at $1090 \mathrm{~mm}, 1150 \mathrm{~mm}$, etc., show longer delay times in accordance with the weaker shock from the remaining particles. Experiment 2 may be the more consistent set, as its jet appears to have been straight, even though offset from the experiment centerline. The mean time of arrival for Experiment 2 is about $1 \mu \mathrm{s}$ earlier than the CALE points, although these times are subject to uncertainties of about $1 \mu \mathrm{s}$ in simulating the calibration of the pins. Interpreting these as differences in the wave velocity is problematical, because this part of the equation of state, below about $2 \mathrm{GPa}$, is essentially unvalidated by the available data.

The $\mathrm{PVF}_{2}$ data are similar to the crystal pin data, except with greater delays. Of the four gauges installed, signals were recorded only from the two that were the most sensitive, and for these the signal-to-noise ratio is small. For the gauge at $1150 \mathrm{~mm}$, the time difference is 42.5 $\mu \mathrm{s}$, and for the gauge at $1270 \mathrm{~mm}$, the time difference is $34.6 \mu \mathrm{s}$. The results are somewhat inconsistent, as one would have expected the shorter time delay to be associated with the earlier pin. However, the signal shape may indicate response to the ionization associated with the passage of the jet. The derived pressures of 0.055 and $0.040 \mathrm{GPa}( \pm 20$ percent) are consistent with expectations from the CALE calculations for similar locations behind preceding foil switches. 


\section{CONCLUSIONS}

The experiments were successful in providing a record of the dynamic hole growth, and the hydrodynamic simulation aided in understanding the phenomena associated with particulated penetration.

The 40-mm-thick sand target provided sufficient contrast on the $450-\mathrm{keV}$ flash radiographs to make the target interaction clearly visible. In particular, the constrictions that develop bet $\mathrm{g} /$ cween the chambers that result from individual particles were revealed. Where longer intervals occur between succeeding particles, more target material remains near the axis. This is shown both in the radiographs and in the simulation. Where the jet is not well aligned, there is a potential for interference with later particles. This was observed in Experiment 1, which had a bent section extending from the tip back to a velocity of $8.2 \mathrm{~km} / \mathrm{s}$, and for which the jet from 8.2 to $7.8 \mathrm{~km} / \mathrm{s}$ did not contribute to penetration.

The CALE calculation satisfactorily reproduced the observed dynamic target cavity growth with a few reasonable assumptions. The axial growth of the hole was matched at the times of the radiographs to 0.5 percent in depth and 1.5 percent in time by using a sand density of 1.60 $\mathrm{g} / \mathrm{cm}^{3}$ and a copper density of $8.4 \mathrm{~g} / \mathrm{cm}^{3}$. The particles were modeled as rods with lengths shortened front and rear by $0.4 \mathrm{~mm}$ to account for tapering. The growth of the hole in radius was also matched to 5 percent by the choice of a maximum yield strength of $2.4 \mathrm{GPa}$ for the sand, combined with a compaction curve that fits the Hugoniot data. The two jets differed in the details of their particle distribution, and we recommend radiography of the actual jet that makes the hole being studied.

The use of foil switches on axis together with piezoelectric crystal pins and $\mathrm{PVF}_{2}$ pressure gauges off axis permitted comparisons to be made between the compaction wave propagation in the experiments and the simulation. We observed agreement within $1 \mu \mathrm{s}$, but closer comparison would require better information about the instrument response functions and about the low-pressure end of the compaction curve.

\section{Acknowledgments}

The authors wish to acknowledge the engineering efforts of Donald S. Bretl, Erni F. Halaxa, Ronald M. Hamilton, Bret E. Knapp, and Denise M. Kuklo. This work was supported in part by the Joint DoD/DOE Munitions Technology Development Program. Work performed under the auspices of the U.S. Department of Energy by the Lawrence Livermore National Laboratory under contract No. W-7405-ENG-48.

\section{References}

Held, M. 1987, Proc. Tenth International Ballistics Symposium, San Diego, Calif., Vol. 2.

Hofmann, R., Andrews, D. J., and Maxwell, D. E. 1968, J. Appl. Phys., 39, 4555-4562.

Reaugh, J. E. 1987, J. Appl. Phys., 61, 962-968.

Tipton, R. E. 1995, CALE User's Manual, private communication.

Van Thiel, M. 1977, Compendium of Shock Wave Data, Lawrence Livermore National Laboratory, UCRL-50108, Rev. 1. 